\title{
Danke, Lissabon?! Die Integrationsverantwortung der Landesparlamente
}

Europa befindet sich am Scheideweg: Es steht zwischen Staatenbund und Bundesstaat. ${ }^{1}$ Daraus ergeben sich erhebliche Schwierigkeiten bei der Abgrenzung von Zuständigkeiten innerhalb dieses mehrfach föderal strukturierten Gebildes von den Bundesländern über die Bundesrepublik bis hin zur Europäischen Union. ${ }^{2}$ Virulent wird die Komplexität der Thematik durch ein Spannungsfeld von neuen Kompetenzen durch den Lissabon-Vertrag sowie den gleichzeitigen Verlust von originären Kompetenzen im Bereich der Landtage. Denn die Landesparlamente werden zunehmend entmachtet ${ }^{3}$ und scheinen die Verlierer einer zunehmenden europäischen Integration zu sein.

Der Aufsatz beschäftigt sich mit den Konsequenzen, die sich aus dem Lissabon-Urteil $^{4}$ des Bundesverfassungsgerichts im Hinblick auf die Einflussmöglichkeiten der Landesparlamente bei der Gesetzgebung der Europäischen Union (EU) ergeben.

Zunächst wird die formal wichtigste Kompetenzausübungsschranke bei der unionsrechtlichen Gesetzgebung, das so genannte Subsidiaritätsprinzip aus Art 5 III EUV, dargestellt und gezeigt, wie die Rechtsprechung des Europäischen Gerichtshofs (EUGH) diese Schranke ausgelegt hat (I.). Danach werden die Auswirkungen des Lissabon-Urteils als auch der Verträge der Europäischen Union auf die Integrationsverantwortung ${ }^{5}$ der Landesparlamente aufgezeigt (II.). Anschließend werden die Forderungen aus der Integrationsverantwortung dargestellt (III.) und der momentane Stand bei der Umsetzung der Integrationsverantwortung am Beispiel des Hessischen Landtages gezeigt (IV.). Danach wird ein Fazit gezogen (V.).

\section{Das Subsidiaritätsprinzip}

Das Urteil des Bundesverfassungsgerichtes ist nicht vom Himmel gefallen, es stellt vielmehr eine Reaktion auf die immer weiter gehende Erstreckung der Unionsgesetzgebung auf Bereiche der Bundes- als auch insbesondere der Landeskompetenz dar. Mit der Verlagerung einher geht eine Entparlamentarisierung, da die Mitwirkung im Rahmen der Europäischen Union durch die Exekutive, nämlich durch die Bundes- und die

1 E. G. Heidbreder, Deutsche EU-Kritik im Lissabon-Zeitalter, EurActiv.de-Debatte vom 11.8.2010.

2 Siehe dazu H.-J. Papier, Zur Verantwortung der Landtage für die europäische Integration, in: ZParl, 2010, S. 903-908.

3 C. Grimm, Landesparlamente im Mehrebenensystem, in: Die Zukunft des Föderalismus in Deutschland und Europa, D. Merten, Berlin, 2007, S. 185-207; H.-J. Papier, Zur Verantwortung der Landtage für die europäische Integration, in: ZParl, 2010, S. 903-908.

4 BVerfG NJW, 2009, S. 2267.

5 Die vom Bundesverfassungsgericht in seinem Urteil hervorgehobene Integrationsverantwortung meint die positive Steuerungs- und Legitimationsfunktion der nationalen Parlamente bei der Gestaltung und dem Erlass von Unionsrecht; W. Kluth, Die Integrationsverantwortung der Landesparlamente, in: LKV, 2010, S. 302-306. 
Landesregierungen wahrgenommen wird. ${ }^{6}$ Vielfach ist deshalb die Rede vom Exekutivföderalismus. ${ }^{7}$ Die Hochzonung von Kompetenzen hat zur Verlagerung eben dieser von den unmittelbar demokratisch legitimierten Parlamenten auf die Exekutive geführt. ${ }^{8}$ Dies ist gerade im Hinblick auf die Auslegung der Subsidiaritätsklausel durch den Europäischen Gerichtshof sehr bedenklich, soll gerade diese doch als Kompetenzausübungsschranke ${ }^{9}$ bei der Verlagerung der Aufgaben dienen.

Das Subsidiaritätsprinzip ist nicht neu. Das durch den Maastrichter Vertrag eingeführte Prinzip ist nun in Art. 5 III AEUV expressis verbis normiert und besagt, dass die Europäische Union bei Vorliegen einer geteilten Zuständigkeit gemäß Art. 4 AEUV unter zwei kumulativen Voraussetzungen handeln darf: ${ }^{10}$

- Die Unionsziele können durch die Mitgliedstaaten und ihre Untergliederungen nicht ausreichend verwirklicht werden und

- die Unionsziele sind wegen ihres Umfangs oder ihrer Wirkungen auf Unionsebene besser zu verwirklichen.

Vorangestellt sei, dass der Europäische Gerichtshof bisher noch nie einen Verstoß gegen das Subsidiaritätsprinzip festgestellt hat. Trotz der formal strengen Voraussetzungen, die mittlerweile unumstritten justiziabel sind, gibt es doch in der Praxis erhebliche Schwierigkeiten. ${ }^{11}$

In der Entscheidung zur Kabelerweiterung von Programmen erklärte der Gerichtshof, dass sich ein Mitgliedstaat durch das Subsidiaritätsprinzip seinen Verpflichtungen aus einer Richtlinie nicht entziehen könne. ${ }^{12}$ Nach dieser Auslegung hätte Sekundärrecht Vorrang vor dem primärrechtlich verankerten Subsidiaritätsprinzip. In die gleiche Richtung geht die zweite Entscheidung zur Tabakwerberichtlinie, in der es zwar nicht um die Subsidiarität, sondern um die Kompetenzausübungsschranke des Gesundheitsschutzes ging. Dort stellte der Europäische Gerichtshof fest, dass es ausreicht, wenn die Tatbestandsvoraussetzungen einer Kompetenzgrundlage, hier die Harmonisierungsvorschrift aus Art 95 EG a.F., vorliegen, obwohl das Gericht bescheinigte, dass dem Gesundheitsschutz ein maßgeblicher Einfluss im vorliegenden Fall zukam. ${ }^{13}$ Nach dieser Auffassung käme den Kompetenzausübungsschranken keine Bedeutung zu, zumindest aber nicht als Schranke bei der Kompetenzausübung. Im Urteil zur Biopatentrichtlinie reichte es, dass die Begründungserwägungen „stillschweigend, aber offenkundig“ auf

6 R. Holtschneider, Ist das Ende des Föderalismus im Europäischen Staatenbund erreicht?, in: DÖV, 2009, S. 1018-1027, K. F. Gärditz/C. Hillgruber, Volkssouveränität und Demokratie ernst genommen - Zum Lissabon-Urteil des BVerfG, in: JZ, 2009, S. 872-881, H.-J. Papier, Zur Verantwortung der Landtage für die europäische Integration, in: ZParl, 2010, S. 903-908.

7 P. Dann, Parlamente im Exekutivföderalismus, Berlin, 2003, S. 4; C. Grimm, Landesparlamente im Mehrebenensystem, in: Die Zukunft des Föderalismus in Deutschland und Europa, D. Merten, Berlin, 2007, S. 185-207.

8 H.-J. Papier, Zur Verantwortung der Landtage für die europäische Integration, in: ZParl, 2010, S. 903-908.

9 A. Haratsch/C. Koenig/M. Pechstein, Europarecht, Tübingen, 7. Aufl. 2010, Rn. 167.

10 Siehe zum Subsidiaritätsprinzip A. Haratsch/C. Koenig/M. Pechstein, Europarecht, Tübingen, 7. Aufl. 2010, Rn. 164 ff.

11 R. Streinz, Europarecht, 8. Auflage, München, 2008, Rn. 166.

12 EuGH Rs. C-11/95, Rn. 53.

13 EuGH Rs. C-380/03, Rn. 92, 96. 
die Beachtung des Subsidiaritätsprinzips eingingen. ${ }^{14}$ Alles in allem zeigt sich, dass das Subsidiaritätsprinzip als Kompetenzausübungsschranke in der Praxis kaum eine Rolle spielt. Dadurch werden Kompetenzen zur Kalamität. ${ }^{15}$

Das Subsidiaritätsprinzip hat ebenso wie die Erforderlichkeitsklausel im Grundgesetz keinen wirksamen Schutz der niedrigeren Regelungsebene gewähren können. ${ }^{16}$

Nur am Rande erwähnt sei das sogenannte Spiel über die Bande. Hintergrund ist ein strategischer Ansatz: Kompetenzen werden in bestimmten Bereichen, in denen Vorhaben auf nationaler Ebene nicht durchsetzbar sind, auf die Europäische Union übertragen, um so verwirklicht zu werden. ${ }^{17}$ Das Ergebnis ist ebenfalls ein nationaler Kompetenzverlust.

\section{Auswirkungen des Vertrages von Lissabon und der neuen Rechtsprechung auf die} Integrationsverantwortung der Landesparlamente

\section{Die Verträge der Europäischen Union}

a) Ausgangspunkt für die Grundlage der Integrationsverantwortung ist die Bedeutung des Begriffs der Subsidiarität, der im EU-Vertrag verankert ist und im Wesentlichen auf die katholische Soziallehre ${ }^{18}$ rückführbar ist. In dem Aufsatz „Das Prinzip der Subsidiarität aus der Sicht der vergleichenden Verfassungslehre" von Häberle ${ }^{19}$ werden die immanenten, also ungeschriebenen Bezugnahmen auf das Subsidiaritätsprinzip dargestellt. So ist der Föderalismus ${ }^{20}$ eine subsidiaritätsnahe Organisationsform.

Ein wesentlicher Bestandteil des Föderalismus ist die vertikale Gewaltenteilung und somit heute eine der rechtspolitischen Begründungen für das Bestehen des Föderalismus in Deutschland. Vertikal kann eine solche aber nur dann sein, wenn sie von gleichen Gewalten wahrgenommen wird, ansonsten würde die Grenze zur horizontalen Gewaltenteilung verwischen. Deshalb müssten die Kompetenzen der Mitwirkung und die

14 EuGH Rs. C-377/98, Rn. 33.

15 J. Wuermeling, Kalamität Kompetenz: Zur Abgrenzung der Zuständigkeiten in dem Verfassungsentwurf des EU-Konvents, in: EuR, 2004, S. 216-229.

16 H.-J. Papier, Zur Verantwortung der Landtage für die europäische Integration, in: ZParl, 2010, S. 903-908.

17 R. Herzog, Die EU schadet der Europa-Idee, FAZ vom 15.1.2010, scharf formuliert von $J$. Wuermeling, Kalamität Kompetenz: Zur Abgrenzung der Zuständigkeiten in dem Verfassungsentwurf des EU-Konvents, in: EuR, 2004, S. 216-229: „Nirgends ist ein Minister freier als hinter den verschlossenen Türen des Ministerrats. In einigen Fällen diente die prestigeträchtige Beauftragung Europas mit einer Aufgabe als Alibi, das die Mängel der nationalen Politik überdecken sollte. Oft erhofften sich die Mitgliedstaaten, durch den Kompetenztransfer unpopuläre Maßnahmen besser durchzusetzen oder politischen Widerstand zu Hause auszuschalten. Und oft geht es einfach nur um weitere europäische Finanztransfers oder gar nur um Symbolik."

18 C. Ritzer/M. Rutloff, Die Kontrolle des Subsidiaritätsprinzips: Geltende Rechtlage und Reformperspektiven, in: EuR 2006, S. 116-137.

19 P. Häberle, Das Prinzip der Subsidiarität aus der Sicht der vergleichenden Verfassungslehre, in: AöR, Bd. 119, S.169-206.

20 P. Häberle, Das Prinzip der Subsidiarität aus der Sicht der vergleichenden Verfassungslehre, in: AöR, Bd. 119, S.169-206. 
Kontrolle der Gesetzgebung der Europäischen Union nicht allein durch den Bundesrat, in dem nur die Vertreter der Länderexekutive sitzen, sondern auch von den Landtagen wahrgenommen werden können. Nur dann wird die vertikale Gewaltenteilung ihrem Namen gerecht, indem die gesetzgebende Gewalt von der gesetzgebenden Gewalt auf tieferer Ebene kontrolliert wird, indem dieser Gewalt eigene Zuständigkeiten zugewiesen sind. Diese Kompetenzverteilung ist im Rahmen der Subsidiarität von der höheren Ebene zu achten.

b) Ebenso gehört nach Häberle die Demokratie als bürgernahe Staatsform zu den immanenten Bezugnahmen auf die Subsidiarität. Nach Art. 2 EUV gehört die Demokratie auch zu den Grundwerten, auf die die Union verpflichtet wird, weshalb die nationalen Parlamente nach Art. 12 EUV eine besondere Bedeutung für die Verwirklichung in Europa haben. Aus dem Demokratieprinzip folgt eine Achtung der Kompetenzen der unmittelbar demokratisch legitimierten Landtage.

c) Weiterhin hat die Union nach Art. 4 II 1 EUV die nationale Identität zu achten, die in den grundlegenden politischen und verfassungsmäßigen Strukturen des Mitgliedstaats zum Ausdruck kommt. Dazu zählt in der bundesstaatlich organisierten Bundesrepublik Deutschland sowohl die Verbands- als auch die Organkompetenz. Betrifft ein europäischer Rechtsakt die Gesetzgebung auf Länderebene, wäre auch der Landtag und nicht nur die Landesexekutive zu beteiligen. Schließlich werden die nationalen Parlamente und nicht die Regierungen von der Europäischen Union in ihrem Protokoll über die Rolle der nationalen Parlamente in der Europäischen Union und auch in die Überprüfung der Einhaltung der Subsidiarität durch das Protokoll über die Anwendung der Grundsätze der Subsidiarität und der Verhältnismäßigkeit eingebunden.

\section{Lissabon-Urteil}

Das Urteil des Zweiten Senats des Bundesverfassungsgerichts verlangte insbesondere eine Stärkung der Mitwirkung des Bundestages als unmittelbar demokratisch legitimiertes Bundesorgan. ${ }^{21}$ Zur Integrationsverantwortung der Landesparlamente wird nichts ausdrücklich erwähnt. Vielmehr geht es im Urteil um das verfassungsrechtliche Gebot der Mitwirkung von Bundesrat und Bundestag. Inhaltlich widmet sich das Urteil der Vertragserweiterung und -ergänzung wie bei einem förmlichen Ratifikationsverfahren. In einem solchen Kontext kann das Urteil nicht unmittelbar auf die Landesparlamente Bezug nehmen. ${ }^{22}$ Auch Art. 23 GG als Ermächtigungsgrundlage für die Übertragung von Hoheitsrechten gesteht die Mitwirkungsbefugnisse nicht den Landtagen, sondern dem Bundesrat zu, der mit Vertretern der Landesregierungen besetzt ist. Dies bedeutet jedoch nicht, dass die Landtage keine Adressaten des Urteils sind.

21 BVerfG, NJW 2009, S. $2271 \mathrm{f}$.

22 H.-J. Papier, Zur Verantwortung der Landtage für die europäische Integration, in: ZParl, 2010, S. 903-908. 
a) Das Bundesverfassungsgericht geht in seinem Urteil von einem integrationsfesten Kern $^{23}$ aus. „Die europäische Vereinigung auf der Grundlage einer Vertragsunion souveräner Staaten darf allerdings nicht so verwirklicht werden, dass in den Mitgliedstaaten kein ausreichender Raum zur politischen Gestaltung der wirtschaftlichen, kulturellen und sozialen Lebensverhältnisse mehr bleibt.“24

$\mathrm{Zu}$ diesem integrationsfesten Kern zählt das Bundesverfassungsgericht ${ }^{25}$,die Staatsbürgerschaft, das zivile und militärische Gewaltmonopol, Einnahmen und Ausgaben einschließlich der Kreditaufnahme sowie die für die Grundrechtsverwirklichung maßgeblichen Eingriffstatbestände, vor allem bei intensiven Grundrechtseingriffen wie dem Freiheitsentzug in der Strafrechtspflege oder bei Unterbringungsmaßnahmen. Zu diesen bedeutsamen Sachbereichen gehören auch kulturelle Fragen wie die Verfügung über die Sprache, die Gestaltung der Familien- und Bildungsverhältnisse, die Ordnung der Meinungs-, Presse und Versammlungsfreiheit oder der Umgang mit dem religiösen oder weltanschaulichen Bekenntnis.“

Viele dieser Kompetenzen sind Landeskompetenzen. ${ }^{26}$ Grundrechtsintensive Eingriffstatbestände lassen sich gerade im Polizeirecht finden. Kulturelle Angelegenheiten fallen nach Art. 30, 70 GG grundsätzlich in die Länderkompetenz, dazu gehören auch die Sprache, die Bildung oder die Pressefreiheit, um nur ein paar Kompetenzen zu nennen. Mithin liegt die Integrationsverantwortung unübertragbar bei den Landesparlamenten, denen innerstaatlich diese Kompetenzen zugewiesen sind. Die Mitwirkungsbefugnisse nach Art. 23 VI und VII GG vermögen einen Kompetenzverlust in diesem Bereich nicht zu kompensieren, schließlich sitzen im Bundesrat Vertreter der Landesregierungen, denen diese Kompetenzen nicht zugewiesen sind. Zwar ist die Verbandskompetenz des Bundesrates gegeben, jedoch nicht die Organkompetenz. Generell stellt die Hochzonung der Kompetenzen eine Verlagerung von eigenständiger landespolitischer Gestaltungsfreiheit hin zu einer bloßen Beteiligung an Entscheidungen des Bundes dar. ${ }^{27}$ Dies ist als aliud keine Kompensation für den Verlust dieser originären Kompetenzen.

b) Zudem gehört auch die Eigenstaatlichkeit der Länder als Ausprägung des in Art. 20 und 79 GG niedergelegten Bundesstaatsprinzips über Art. 23 I 3 GG zu den Grenzen einer europäischen Integration. Den Ländern muss deshalb ,ein Kern eigener Aufgaben unentziehbar verbleiben. “28

23 M. Ruffert, An den Grenzen des Integrationsverfassungsrechts: Das Urteil des Bundesverfassungsgerichts zum Vertrag von Lissabon, in: DVBl, 124. Jg. (2009), S. 1197-1208.

24 BVerfG, NJW 2009, S. 2273.

25 BVerfG, NJW 2009, S. 2274.

26 Siehe dazu auch H.-J. Papier, Zur Verantwortung der Landtage für die europäische Integration, in: ZParl, 2010, S. 903-908.

27 C. Grimm, Landesparlamente im Mehrebenensystem, in: Die Zukunft des Föderalismus in Deutschland und Europa, D. Merten, Berlin, 2007, S. 185-207.

28 BVerfGE 34, 9, 20. 
c) In die gleiche Richtung geht die vom Bundesverfassungsgericht angemahnte UltraVires-Kontrolle unionsrechtlicher Hoheitsakte. ${ }^{29}$ Demnach prüft das Bundesverfassungsgericht, wenn Rechtschutz auf Unionsebene nicht zu erlangen ist, ob Rechtsakte europäischer Organe und Einrichtungen sich unter Wahrung des gemeinschafts- und unionsrechtlichen Subsidiaritätsprinzips in den Grenzen der ihnen im Wege der begrenzten Einzelermächtigung eingeräumten Hoheitsrechte halten. Die Integrationsverantwortung bezüglich der Kompetenzen, die vormals innerstaatlich den Ländern zugeordnet waren, trägt auch der Landtag. Die Integrationsverantwortung der Landesparlamente könnte also je nach Ausgestaltung eine prozedurale Ergänzung zur Ultra-ViresKontrolle des Bundesverfassungsgerichts darstellen.

d) $\mathrm{Zu}$ den Integrationsgrenzen der Staatlichkeit und der Unverfügbarkeit der verfassungsgebenden Gewalt ${ }^{30}$ kommt die inhaltliche Schranke des Demokratieprinzips, die durch Art. 23 I 1 und 3 GG abgesichert ist.

Zum Demokratieprinzip gehört, dass alle Gewalt von den unmittelbar demokratisch legitimierten Parlamenten auszugehen hat. Dadurch wird impliziert, dass der Bürger erkennen kann, von wem seine Rechte wahrgenommen werden. Durch eine gestreckte Legitimationskette verliert er die Möglichkeit durch seinen Wahlakt, die Grundlinien der Politik zu bestimmen. ${ }^{31}$

Legitimation kann auch gemittelt werden. Dieser Hilfe bedürfte es nicht, wäre das Europäische Parlament selbst hinreichend demokratisch legitimiert. Ohne Zweifel sind die Zuständigkeiten des Europäischen Parlaments durch den Vertrag von Lissabon ausgeweitet worden, was sehr zu begrüßen ist, allerdings ,,betont das Bundesverfassungsgericht, dass selbst zusammengenommen das Europäische Parlament mangels eines einheitlichen Unionsvolkes und wegen der daraus resultierenden Ungleichheiten in der Ausgestaltung des Wahlrechts einerseits und der Europäische Rat bzw. Ministerrat als rein exekutiv zusammengesetzte Organe der Europäischen Union andererseits der Union alleine keine ausreichende demokratische Legitimation vermitteln können und deswegen den nationalen Parlamenten eine wichtige Rolle zukommt. "32 Insofern kommt es auf die hinreichende Vermittlung der demokratischen Legitimation über die nationalen Parlamente an. ${ }^{33}$

29 BVerfG, NJW 2009, S. 2272.

30 M. Ruffert, An den Grenzen des Integrationsverfassungsrechts: Das Urteil des Bundesverfassungsgerichts zum Vertrag von Lissabon, in: DVBl, 124. Jg. (2009), S. 1197-1208.

31 C. Grimm, Landesparlamente im Mehrebenensystem, in: Die Zukunft des Föderalismus in Deutschland und Europa, D. Merten, Berlin, 2007, S. 185-207.

32 C. D. Classen, Legitime Stärkung des Bundestages oder verfassungsrechtliches Prokrustesbett, in: JZ, 2009, S. 881- 889; BVerfG, NJW 2009, S. 2280.

33 So auch P. Dann, Parlamente im Exekutivföderalismus, Berlin, 2003, S. 6, der aber auf Seite 164 meint, dass der Verlust an Kompetenzen dadurch kompensiert werde, dass den Gliedstaaten der Vollzug des Rechts vorbehalten und ihnen andererseits Mitwirkungsrechte erhalten bleiben. „Die Legitimation durch nationale Parlamente steht unter dem Vorbehalt exekutivföderaler Strukturen.“ Auf Seite 269 heißt es dann weiter: „Effektive nationalparlamentarische Legitimation und exekutivföderale Institutionenordnung konterkarieren sich.“ 
Der Bundestag kann nur dort demokratische Legitimation unmittelbar vermitteln und übertragen, wo er eine innerstaatliche Kompetenz hat. Andernfalls mittelt auch er lediglich die unmittelbare Legitimation.

Es geht hier folglich um die Suche nach der Wurzel der Legitimation, um zu prüfen, ob der Legitimationsstrang noch nah genug am Souverän ist.

Demokratische Legitimation geht im Bereich der Länderkompetenzen unmittelbar von den Landtagen aus. Mitwirkungsbefugnisse des Bundesrates bei der Übertragung von Hoheitsrechten nach Art. 23 VI und VII GG stellen zwar eine demokratische Legitimation in diesem Bereich dar, allerdings wiederum nur mittelbar. Denn die Landesregierungen sind von den Landtagen gewählt und die Organkompetenz liegt ausschließlich bei den Parlamenten.

Es ergibt sich also bezüglich der demokratischen Legitimation bei Übertragung von Hoheitsrechten, die in Länderkompetenz stehen, folgendes Bild: Die Legitimation vollzieht sich somit vom Landtag über die Landesregierung zu den deutschen Vertretern der Exekutive im Ministerrat bzw. im Europäischen Rat.

Insofern erscheint diese Legitimation sehr schwach, ${ }^{34}$ auch wenn man zu Recht wie das Bundesverfassungsgericht berücksichtigt, dass Art. 23 I 1 GG nicht eine vollkommene Gleichstellung mit dem Demokratieprinzip aus Art. 20 und 28 GG verlangt. Es sind vielmehr auch die Traditionen anderer Mitgliedstaaten sowie die Besonderheiten der Struktur eines Staatenverbundes zu berücksichtigen. ${ }^{35}$

Dies besagt, dass die Landesparlamente auch Trägerinnen der Integrationsverantwortung sind. Sie sind die Wurzel der demokratischen Legitimation und müssen deshalb auf Grund der Länge der demokratischen Legitimation einen Teil der Verantwortung tragen können. Es geht nicht darum die Exekutive zu schwächen, es geht vielmehr darum die Legislative zu stärken, um gemeinsam die Verantwortung wahrzunehmen. Mit den Worten von Ruffert, ${ }^{36}$ der seine Ausführungen allerdings auf die nationalen Parlamente bezieht: „Mithin können sich die mitgliedstaatlichen Organe der Verantwortung für die Einhaltung zentraler Prinzipien im Integrationsprozess nicht begeben, und vor allem die [nationalen] Parlamente müssen die entscheidenden Schritte der Integration mit den ihnen zur Verfügung stehenden Mitteln begleiten.“

e) Ebenso wird über Art. 23 I 3 i.V.m. Art. 79 III GG die demokratische Legitimation der Landesstaatsgewalt geschützt. Durch die Übertragung von Aufgaben auf die Europäische Union, mit der eine Entstaatlichung des Gesamtstaates und Entdemokratisierung einhergehen, würde die Landesstaatsgewalt ebenfalls entwertet. ${ }^{37}$ Dies gilt aber auch

34 Siehe insgesamt zu den Legitimationsketten in der EU C. Henke, Plädoyer für kürzere Legitimationsketten in der Europäischen Union, in: EuR, 2010, S.118-137; ebenfalls mit Plädoyer zu kürzeren Legitimationsketten: C. Grimm, Landesparlamente im Mehrebenensystem, in: Die Zukunft des Föderalismus in Deutschland und Europa, D. Merten, Berlin, 2007, S. 185-207.

35 C. D. Classen, Legitime Stärkung des Bundestages oder verfassungsrechtliches Prokrustesbett, in: JZ, 2009, S. 881- 889.

36 M. Ruffert, An den Grenzen des Integrationsverfassungsrechts: Das Urteil des Bundesverfassungsgerichts zum Vertrag von Lissabon, in: DVB1, 124. Jg. (2009), S. 1197-1208.

37 K. F. Gärditz/C. Hillgruber, Volkssouveränität und Demokratie ernst genommen - Zum Lissabon-Urteil des BVerfG, in: JZ, 2009, S. 872-881. 
hinsichtlich der Gewaltenteilung, ,soweit der Europäisierungsprozess zu Verschiebungen in der staatsstrukturellen Tektonik führt, die die Funktionsfähigkeit der Legislative als Basis demokratischer Legitimation erodieren." 38 Es wird folglich über Art. 23 I 3 i.V.m. Art. 79 III GG die Gewaltenteilung geschützt, so dass auch aus dieser bei der Übertragung von Hoheitsrechten an die Europäische Union den Landesparlamenten eine Integrationsverantwortung erwächst.

\section{Die Forderungen aus der Integrationsverantwortung}

Um die Integrationsverantwortung wirksam wahrzunehmen, bedarf es einer Absicherung der Kompetenzen, wenn sie nicht leer laufen sollen. Kompetenz bedeutet gerade die eigenverantwortliche Wahrnehmung von Aufgaben. Aus einer Zuständigkeit müssen abwehrfähige Rechtsgüter erwachsen. Die eigenverantwortliche Wahrnehmung der Rechtsgüter muss prozedural und/oder prozessual geschützt werden.

Dazu werden folgende Vorschläge vertreten:

- Ein eigenständiges Klagerecht vor dem Bundesverfassungsgericht mit einer Klarstellung, dass das Klagerecht auch binnenverfassungsrechtlicher Natur ist (a).

- Ein Weisungsrecht gegenüber der Landesregierung (b).

- Die Stärkung der Informationsrechte (c).

a) „Das verfassungsprozessuale Rechtsschutzsystem ist auf den Schutz des Vorrangs der Verfassung gegenüber nachrangigen nationalem Recht (Art 20 III GG, Art 1 III GG, Art 93 I Nr. 2, Nr. 4 a. Nr. 4 b GG) oder auf den Vorrang des Bundesrechts vor dem Landesrecht (Art 93 I Nr. 2 GG) sowie auf die Einhaltung der bundesstaatlichen Kompetenzverteilung im Rahmen des Gewaltenteilungsprinzips (Art. 93 I Nr.1 GG) zugeschnitten. "39 Insofern kommt die Normierung eines eigenständigen Klagerechts vor dem Bundesverfassungsgericht auch für die Landesparlamente in Betracht.

Von einer solchen Normierung könnte allerdings eine negative Signalwirkung für die Rechtseinheit in der EU und eine Eskalationsgefahr ausgehen. ${ }^{40}$ Es besteht die Gefahr, dass die Kommission gegen diese Normierung ein Vertragsverletzungsverfahren einleitet und es zu einem Kampf um die Kompetenzen zwischen Europäischem Gerichtshof und Bundesverfassungsgericht kommt. Dem kann man entgegenhalten, dass die Landtage nicht auf ihre Rechte verzichten dürfen, weil mit ihrer Ausübung Probleme verbunden sein können.

Die Normierung eines Klagerechts der Landtage könnte aber zu Parallelprüfungen führen, wenn der Bundestag oder der Bundesrat eine Subsidiaritätsklage vor dem Europäischen Gerichtshof erhebt, während ein Landesparlament vor dem Bundesverfassungsgericht klagt. Eine klare gesetzliche Ausgestaltung kann aber auch in solchen

38 K. F. Gärditz/C. Hillgruber, Volkssouveränität und Demokratie ernst genommen - Zum Lissabon-Urteil des BVerfG, in: JZ, 2009, S. 872-881.

39 H. A. Wolff, De lege ferenda: Das Integrationskontrollverfahren, in: DÖV, 2010, S. 49-58; siehe auch K. F. Gärditz/C. Hillgruber, Volkssouveränität und Demokratie ernst genommen - Zum Lissabon-Urteil des BVerfG, in: JZ, 2009, S. 872-881.

40 H. Sauer, Kompetenz- und Identitätskontrolle von Europarecht nach dem Lissabon-Urteil, in: ZRP, 2009, S. 195-198. 
Fragen Abhilfe schaffen. ${ }^{41}$ Eine Normierung bringt vielmehr Rechtssicherheit und kann sogar derart gestaltet werden, dass weniger verfassungsgerichtliche Kontrolle zugelassen wird als bislang möglich. ${ }^{42}$

Ebenfalls spricht für die Landtage als Klageberechtigte, dass sie an dem langwierigen Entscheidungsprozess der unionsrechtlichen Gesetzgebung nicht beteiligt sind und auf dieser Ebene niemand, zumindest unmittelbar, für die Interessen der Landesparlamente eintritt.

Somit ergibt sich, dass trotz berechtigter Bedenken ein Klagerecht der Landtage normiert werden sollte. Eine Erweiterung dieses Klagerechts auf die binnenverfassungsrechtlichen Kompetenzen der Landtage ist dabei nur stringent.

b) Ein weiteres Mittel, um die Durchsetzung der Integrationsverantwortung der Landesparlamente zu sichern, könnte ein Weisungsrecht der Landesparlamente gegenüber den Landesregierungen darstellen.

Grundsätzlich ist es ureigenste Aufgabe eines Parlamentes, die Exekutive zu kontrollieren. Allerdings sind die Kontrollmaßnahmen wie etwa Beschlüsse nicht rechtlich verbindlich. „Der Grund hierfür liegt in der Organselbständigkeit der Landesregierung, der gegenüber das Landesparlament keine allgemeine, von gesetzlichen Grundlagen losgelöste Prärogative besitzt." "43 Zudem kann Landesrecht nicht die Bindungen eines Bundesorgans festsetzen, dessen Kompetenzen sich aus dem Grundgesetz ergeben. ${ }^{44}$ Vielmehr geht das Bundesrecht vor. Das Bundesverfassungsgericht hat zudem in seiner Entscheidung zum Zuwanderungsgesetz ${ }^{45}$ festgestellt, dass das Recht, den Vertretern im Bundesrat Weisungen zu erteilen, den Landesregierungen zusteht. Allerdings besteht die Möglichkeit, den Art. 51 GG dahingehend zu ändern, dass ein Weisungsrecht der Landesparlamente gegenüber den Vertretern der Länder im Bundesrat festgeschrieben wird. Dem würden auch keine verfassungsrechtlichen Bedenken entgegenstehen. Ein Verstoß gegen das Bundesstaatsprinzip ist durch eine Änderung nicht ersichtlich. Vielmehr liegt eine bundesrechtliche Regelung zum Organisationsrecht eines Bundesorgans vor. ${ }^{46}$

Als eine Alternative zu dieser Regelung könnte man nach Grimm $^{47}$ auch eine Öffnungsklausel aufnehmen, nach der das Weisungsrecht landesrechtlich ausgestaltet werden soll. Diese Lösung würde den Vorzug haben, dass ein Problem, das die Landesebene betrifft, auch landesverfassungsrechtlich geregelt werden könnte. Diesen Weg ist nun Baden-Württemberg gegangen und hat im Rahmen einer Verfassungsänderung und ei-

41 H. A. Wolff, De lege ferenda: Das Integrationskontrollverfahren, in: DÖV, 2010, S. 49-58.

42 H. Sauer, Kompetenz- und Identitätskontrolle von Europarecht nach dem Lissabon-Urteil, in: ZRP, 2009, S. 195-198.

43 C. Grimm, Landesparlamente im Mehrebenensystem, in: Die Zukunft des Föderalismus in Deutschland und Europa, D. Merten, Berlin, 2007, S. 185-207.

44 Siehe nur C. Grimm, Landesparlamente im Mehrebenensystem, in: Die Zukunft des Föderalismus in Deutschland und Europa, D. Merten, Berlin, 2007, S. 185-207.

45 BVerfGE 106, 310, 334.

46 Siehe hierzu C. Grimm, Landesparlamente im Mehrebenensystem, in: Die Zukunft des Föderalismus in Deutschland und Europa, D. Merten, Berlin, 2007, S. 185 - 207.

47 C. Grimm, Landesparlamente im Mehrebenensystem, in: Die Zukunft des Föderalismus in Deutschland und Europa, D. Merten, Berlin, 2007, S. 185-207. 
nes Gesetzes ein Weisungsrecht manifestiert. ${ }^{48}$ Zur Notwendigkeit der Änderung rekurrieren die Gesetzesmaterialien auf die Fortentwicklung der Europäischen Union. Insoweit schien die bisherige Praxis (seit 1995 wird die Beteiligung des Landtags in Art. 34 a LV BW geregelt) als nicht mehr zeitgemäß.

Die zunehmende Verlagerung der Rechtssetzung auf die Europäische Union habe zu einer Entparlamentarisierung geführt, deren Kompensation nicht durch exekutive Mitspracherechte erfolgen könne. ${ }^{49}$

Dieser Meinung hat sich auch Papier angeschlossen. Er hält die auf Art. 51 GG gestützte Auslegung des Bundesverfassungsgerichts ,für nicht zutreffend“ 50 Die bindende Wirkung von Beschlüssen der Landtage gegenüber der Landesexekutive, sei ausschließlich eine Frage des Landesverfassungsrechts. „Bundesverfassungsrechtliche Vorgaben in diesem Bereich seien sogar grundsätzlich unzulässig. “51

Das EULG präzisiert und verstärkt die Regelung des Art. 34 a LV BW. Es ersetzt die bisherige Vereinbarung zwischen Landtag und Landesregierung. Die Landesregierung wird demnach gestuft an Stellungnahmen des Landtags gebunden: ${ }^{52}$

- Eine strikte Bindung ist vorgesehen, wenn ausschließliche Gesetzgebungszuständigkeiten der Länder ganz oder teilweise auf die EU übertragen werden sollen.

- Eine Bindung, von der aus erheblichen Landesinteressen abgewichen werden kann, soll im Hinblick auf Vorhaben der EU gelten, die im Schwerpunkt ausschließliche Gesetzgebungszuständigkeiten der Länder betreffen.

Die landesverfassungsrechtliche Lösung wirkt somit im Innenverhältnis und lässt das Verhalten der Landesregierung im Außenverhältnis bei der Bundesratsabstimmung unberührt. Mithin zeigt sich, dass diese Lösung ein gangbarer Weg ist, um die Integrationsverantwortung der Landesparlamente zu stärken.

c) Schon jetzt erhalten die Landesparlamente Informationen über den unionsrechtlichen Gesetzgebungsprozess durch die Landesregierungen als auch durch die Bundesorgane. Wichtig wäre hier aber ein eigenes, originäres Informationsrecht der Landesparlamente. Es besteht die Möglichkeit, dass Informationen gefiltert werden und $\S 44$ II GO Bundesrat sieht sogar die Möglichkeit vor, dass Bundesorgane Landesregierungen die Weitergabe von Informationen untersagen können. Vorzuschlagen wäre hier eine gesetzliche Normierung beispielsweise im EUZBLG (Gesetz über die Zusammenarbeit von Bund und Ländern in Angelegenheiten der Europäischen Union), sodass die Landesparlamente Zugriff auf EUDYSIS, die Datenbank über maßgebliche EU-Rechtsetzungsakte, bekommen. Diese Maßnahme kann sicherlich die Landesparlamente stärken und sie ist auch leichter durchsetzbar als die anderen vorgeschlagenen Maßnahmen. Als

48 Gesetz zur Änderung der Verfassung des Landes Baden-Württemberg, Drs. 14/7562, Gesetz zur Beteiligung des Landtags in Angelegenheiten der Europäischen Union (EULG) Drs. $14 / 7339$.

49 Kompensationsargument siehe Abschnitt II. 2. a.

50 H.-J. Papier, Zur Verantwortung der Landtage für die europäische Integration, in: ZParl, 2010, S. 903-908.

51 H.-J. Papier, Zur Verantwortung der Landtage für die europäische Integration, in: ZParl, 2010, S. 903-908.

52 Landtag Baden-Württemberg Drs.14/7339, S. 1. 
einzige Maßnahme würde sie aber der Integrationsverantwortung der Landesparlamente nicht gerecht.

Auf der jährlichen Landtagspräsidentenkonferenz sind die Landtagspräsidenten der Idee der Informationsverbesserung als auch einem Weisungsrecht gegenüber der eigenen Landesregierung näher getreten und haben die sog. „Stuttgarter Erklärung“ einstimmig mit diesem Inhalt verabschiedet. ${ }^{53}$

\section{Stand der Umsetzung am Beispiel des Hessischen Landtages}

Die Landtage sind sich ihrer Verantwortung und der sich daraus ergebenden Chance bewusst. Die Europafähigkeit der Landesparlamente ist momentan stets Thema auf den Präsidenten- und Direktorenkonferenzen.

Jedes Landesparlament hat seine eigene Vorgehensweise, um den europäischen Anforderungen gerecht zu werden. Der Hessische Landtag agiert mit engagierten Parlamentariern und einem eigenen Fachbereich „Europa, Internationale Angelegenheiten“ und versucht, die unterschiedlichsten Informationsstränge für europäische Vorhaben zu nutzen.

Seit dem Jahr 2010 kooperiert er mit der „eigenen“ Landesregierung in EU-Angelegenheiten. Der Ältestenrat des Hessischen Landtags hat am 11. Mai 2010 die vom Europaausschuss vorgelegte ,Vereinbarung über die Unterrichtung des Hessischen Landtages durch die Landesregierung in Angelegenheiten der Europäischen Union“ beschlossen. Das Kabinett hat die Vorlage am 28. Juni 2010 ebenfalls verabschiedet. Danach wird der Landtag unverzüglich schriftlich über alle Vorhaben im Rahmen der Europäischen Union unterrichtet, die für das Land Hessen von erheblicher landespolitischer Bedeutung sind und wesentliche Interessen des Landes unmittelbar berühren, und gibt ihm Gelegenheit zur Stellungnahme. ${ }^{54}$ Als Quasi-Rechtsfolge regelt Nr. 9 der Vereinbarung, dass die Landesregierung ihr rechtzeitig zugegangene Stellungnahmen des Landtags zu Vorhaben der Europäischen Union bei ihrer Entscheidung berücksichtigt, welche die Gesetzgebungszuständigkeiten der Länder wesentlich berühren. Entsprechendes gilt bei der Übertragung von Hoheitsrechten der Länder auf die Europäische Union sowie bei der Entscheidung der Landesregierung im Bundesrat, wenn ein Vorhaben im Schwerpunkt ausschließliche Gesetzgebungsbefugnisse der Länder betrifft und daher die Verhandlungsführung im Rat der Europäischen Union auf einen Vertreter der Länder übertragen worden ist.

Die hausinterne Organisation des Unterrichtungsverfahrens im Parlament sowie in der Verwaltung wird gerade in Absprache mit der Landesregierung erarbeitet. Seit dem 1.1.2011 läuft eine Testphase, um die Zuleitung und das geplante Verfahren zu erproben und zu eruieren. Als Ermächtigungsgrundlage für die interne Landtagsüberweisung dient ein Verfahrenskonzept, das zunächst aus einem Obleutegespräch des Europaausschusses entwickelt und in einem zweiten Schritt durch ein Gespräch des Landtagsprä-

53 „Stuttgarter Erklärung“, u.a. Hessischer Landtag, Drs. 18/2803.; S. Menzenbach, Das Parlament, 20.9.2010.

54 Nr. 1 Satz 1 der Vereinbarung über die Unterrichtung des Hessischen Landtages durch die Landesregierung in Angelegenheiten der Europäischen Union (in der Fassung vom 28. Juni 2010). 
sidenten mit allen Ausschussvorsitzenden und sonstigen Beteiligten weiterkonzipiert wurde. Bedingt durch die operative Dynamik in diesem Verfahrensstadium hat der Hessische Landtag zunächst von einer Geschäftsordnungsänderung abgesehen und sich für ein flexibles Verfahrenskonzept entschieden. Auf dieser Grundlage überweist der Präsident analog §33 GO Hessischer Landtag sämtliche fristgebundenen „Frühwarndokumente" 55 an den Europaausschuss und sonstige Dokumente nach Ziffer 1 insbesondere solche mit erheblicher landespolitischer Bedeutung an die Fachausschüsse zur Beratung. Vor der Überweisung werden die parlamentarischen Geschäftsführer der Fraktionen im Rahmen einer „Ausschussvorschlagsliste“ eingebunden, um die Fachausschussvorschläge der Verwaltung zu legitimieren und gegebenenfalls nicht beratungsrelevante Vorhaben von der Tagesordnung zu nehmen. Bei der Beratung der „Frühwarndokumente“ geht es dem Europaausschuss vornehmlich um die Überprüfung der Einhaltung des Subsidiaritätsgrundsatzes. Im Gegensatz hierzu beschäftigen sich die Fachausschüsse inhaltlich - nicht also nur kompetenzrechtlich - mit den Vorhaben, denen seitens der Landesregierung landespolitische Bedeutung zugemessen wurde. Grundsätzlich entscheiden die Ausschüsse - bedingt durch die zu wahrenden Fristen plenarersetzend. Jedoch gibt es auch die Möglichkeit, dass eine Fraktion innerhalb einer Woche nach Beschlussfassung das Verlangen übermittelt, die Entscheidung des Plenums einzuholen. Diese sog. „Rückhollösung“ basiert entsprechend auf § 33 IV GO Hessischer Landtag.

Als Fazit kann nach einem halben Jahr Testlaufphase saldiert werden, dass der Hessische Landtag über 650 Dokumente zugeleitet bekommen hat. Der Präsident hat davon auf Grund des vorläufigen Verfahrenskonzeptes 40 Vorhaben an die Ausschüsse zur Beratung überwiesen. Daneben hat die Landtagskanzlei eine Datenbank erarbeitet, in der jedes Dokument abrufbar ist, das zugeleitet wurde. Das Verfahren innerhalb des Hessischen Landtags ist demokratisch, effizient und wird den europäischen Vorgaben gerecht.

Unabhängig davon entsendet das Land Hessen derzeit zwei Vertreter in den Ausschuss der Regionen (AdR). Somit erlangt der Landtag frühzeitig Kenntnis über einige Vorhaben im prälegislativen Bereich, da der AdR nach Art. 300 Abs. 1 AEUV gegenüber dem europäischen Parlament, dem Rat und der Kommission beratende Aufgaben wahrnimmt. ${ }^{56}$ Kritisch zu begleiten ist auch in diesem Zusammenhang die Wahrung des Subsidiaritätsprinzips und somit die Einhaltung der europäischen Gesetzgebungskompetenzen. Als aktuelle Indikatoren sind eine beträchtliche Anzahl von Änderungsanträgen heranzuziehen, die Berichte mit einem Hinweis auf Subsidiaritätsverletzung ändern bzw. ablehnen. ${ }^{57}$

55 Dokumente, die im Rahmen des Frühwarnsystems des Art. 12 b EUV durch die EU-Organe an die nationalen Parlamente übermittelt werden, um unmittelbar in den EU-Rechtsetzungsprozess einbezogen zu werden. Siehe auch W. Kaufmann-Bühler, in: Lenz/Borchardt (Hrsg.), EU-Verträge Kommentar, 5. Aufl., Art. 12, Rn. 10.

56 Beratende Aufgaben sind gesetzlich vorgesehen z.B. in Art. 157 III, 166 IV, 168 V AEUV.

57 Genannt seien 12 Änderungsanträge im Rahmen des Plenums vom 04.-06. Oktober 2010, die eine Einhaltung des Subsidiaritätsprinzips anmahnten. 


\section{Fazit}

Den Landesparlamenten kommt eine größere Bedeutung bei einer immer weiter wachsenden europäischen Integrationsverantwortung neben Bundestag und Bundesrat zu. Ihre Stellung im politischen Dialog, im europäischen Rechtsetzungssystem als auch bei der Umsetzung der unionsrechtlichen Rechtsakte in nationales Recht muss gestärkt werden. Die Auslegung des Subsidiaritätsprinzips muss kritisch begleitet werden. Diese Rückkopplung unionsrechtlichen Handelns darf nicht als Last angesehen werden. Vielmehr wird durch eine Beteiligung der unteren Ebenen die Legitimation der europäischen Union auf ein stärkeres Fundament gestellt. Dies stellt eine Chance für eine zunehmende europäische Integration dar. Die Union kann sich dadurch als ein Europa der Bürger bewähren, deren Willen durch die Wahl der Landtage und des Bundestages unmittelbar zum Ausdruck kommt. So kann die Union auch mehr Rückhalt in der Bevölkerung gewinnen. Der Fokus der nationalen Parlamente insbesondere auch der Landtage sollte sich auf den frühestmöglichen Mitberatungszeitpunkt beziehen. Von besonderem Interesse werden deswegen gerade die Grün- und Weißbücher sein, die einen frühen Eintritt in den politischen Dialog bedeuten.

Es bleibt abzuwarten, welchen Weg man auf Landes-, Bundes- und Unionsebene im Hinblick auf die Integrationsverantwortung der Landesparlamente gehen wird. Als Schritt in die richtige Richtung kann man die erste Subsidiaritätsrüge des Bundestages bewerten. ${ }^{58}$

Maßgeblich für den Erfolg der Europafähigkeit der Landtage und deren konsequenter Wahrnehmung der Integrationsverantwortung werden zwei Dinge sein: Erstens, dass die Informationen konsequent zugeleitet werden und zweitens, dass die Informationen in relevantes Wissen umgewandelt und somit verarbeitet werden können. Wichtig ist gerade hier, die genannten Forderungen aus der Integrationsverantwortung perspektivisch im Auge zu behalten.

Als momentanes Fazit für die nationalen Parlamente kann man aber kurz gesagt festhalten: danke Lissabon.

58 Erhoben am 07.10.2010 im Rahmen der Unterrichtung zu dem Vorschlag für eine Richtlinie KOM (2010) 368 EU des Europäischen Parlaments und des Rates über Einlagensicherungssysteme (Neufassung) (inkl. 12386/10 ADD1 und 12386/10 ADD 2) (ADD 1 in Englisch) BT-Drs. 17/2994 Nr. A. 23, 17/3239 -, auch wenn diese das erforderliche Quorum nicht erreicht hat. 